\title{
INFLUENCE OF HYDROSTATIC PRESSURE ON THE YIELD STRENGTH OF ANISOTROPIC POLYCARBONATE
}

\author{
Robert M. CADDELL \\ Department of Mechanical Engineering, The University of Michigan, Ann Arbor, MI 48109,
} U.S.A.

and

\section{Joo Won KiM†}

High Pressure Research Laboratory and Department of Mechanics and Materials Science, Rutgers University, New Brunswick, NJ 08903, U.S.A.

(Received 6 July 1979; in revised form 30 January 1980)

Summary-Tension and compression specimens of anisotropic (oriented) polycarbonate were subjected to hydrostatic pressures up to $552 \mathrm{MPa}(80 \mathrm{ksi})$. The resulting yield behavior was compared with a yield criterion for use with pressure dependent and anisotropic solids. A promising correlation was found and attention is called to certain problems related to the measurement of tensile true stress-true strain behavior during typical hydrostatic pressure testing.

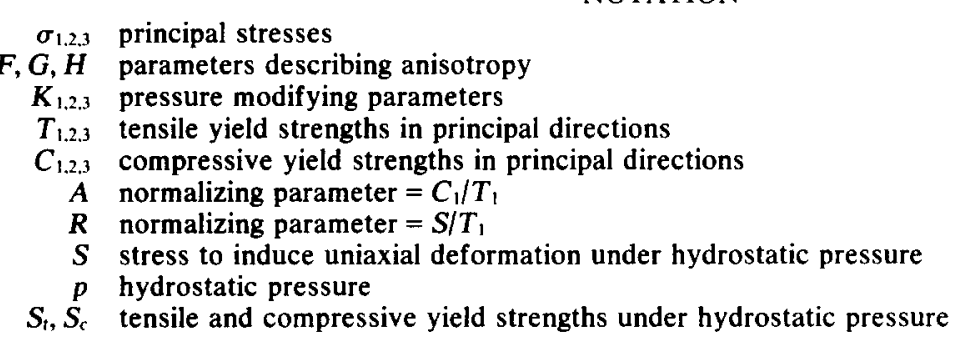

\section{INTRODUCTION}

The influence of the mean normal stress $\ddagger$ upon the mechanical behavior of polymers has been discussed in numerous papers; only certain typical references are noted here. Some [1-3] have summarized the findings of many investigators and contain an extensive and useful list of references. The principal concern in others [4-9] is related to the yield behavior of various polymers. As an aside, it has also been noted that pressure can influence the yield behavior of certain metals [10-12] although, as a general rule, such effects are usually negligible. Since the concern of this paper is with the effect of pressure on the yielding of polymers, no further reference will be made to metals or to other mechanical properties in general.

Findings from many earlier studies [4-9] were compared with a proposed yield criterion [13] and a promising correlation resulted. It would appear that in those studies, unoriented or macroscopically isotropic polymers were used.

To account for anisotropy, as exists with highly oriented polymers, the criterion [13] was modified [14], and subsequent investigations [15, 16] indicated excellent agreement between theoretical predictions and experimental findings. All of these previous studies [13-16] were conducted at atmospheric pressure and a further

†Present address: Hyundai International, Seoul, Korea.

\$This can be altered most readily by a superimposed hydrostatic fluid pressure and the terms mean normal stress and hydrostatic stress are used interchangeably in this context. 
test of this yield criterion[14] was made by observing the influence of large hydrostatic pressures upon the subsequent yield behavior of highly oriented (i.e. anisotropic) polycarbonate (PC). The findings constitute the basis of this paper.

\section{GENERAL APPROACHES IN YIELD STUDIES}

There are various experimental approaches used to compare predictions based upon a proposed yield criterion with results determined by experimentation. Perhaps the most widely used is the development of a yield locus in two-dimensional, principal stress space as typified in particular papers [15,16]. A more complete test of any criterion is related to the development of a yield surface in three-dimensional stress space; to our knowledge, the work of Pae [17] is the only publication of this kind where polymers served as test materials. A third technique is to determine what effect, if any, a hydrostatic pressure at various discrete levels, has upon the subsequent tensile and compressive yield behavior of the material in question. In essence, these results produce two discrete lines that lie on the yield surface. $\dagger$ Another approach may be used when anisotropic sheet material is involved, where the variation of yield strength as a function of angular orientation in the sheet is studied; an example is the work of Caddell et al.[14].

The findings in this paper were based upon the third approach mentioned above wherein tension and compression specimens of highly oriented PC were initially subjected to an all around fiuid pressure. Subsequent loading in one direction was then applied until a specimen yielded, the pressure being maintained throughout a test. All such tests were carried out on a special pressure machine at Rutgers University. The full details of this equipment are given elsewhere [18].

\section{PROPOSED YIELD CRITERION}

The criterion attributed to von Mises [19] is widely used when isotropic, ductile metals are involved. In terms of principal stresses, it is expressed as:

$$
\left(\sigma_{1}-\sigma_{2}\right)^{2}+\left(\sigma_{2}-\sigma_{3}\right)^{2}+\left(\sigma_{3}-\sigma_{1}\right)^{2}=2 T^{2}=2 C^{2}
$$

where $T$ and $C$ are the yield strengths in uniaxial tension and compression respectively. Because it is assumed that $C$ and $T$ are equal and that they are unaffected by the magnitude of the mean normal stress (i.e. independent of pressure), this criterion has been shown to be unsuitable for use with polymers since neither of these key assumptions is substantiated.

To account for these discrepancies, Raghava [20] modified equation (1) for use with isotropic polymers; the form is,

$$
\left(\sigma_{1}-\sigma_{2}\right)^{2}+\left(\sigma_{2}-\sigma_{3}\right)^{2}+\left(\sigma_{3}-\sigma_{1}\right)^{2}+2(C-T)\left(\sigma_{1}+\sigma_{2}+\sigma_{3}\right)=2 C T .
$$

The values of $C$ and $T$ are the absolute values (i.e. signs are neglected) of these respective yield strengths measured at atmospheric pressure; for all polymers they appear to have different values. Pressure effects are introduced by the term containing the sum of the principal stresses. It is noted that others [2,21-23] have either proposed or used a similar or identical form of equation (2); in addition, if $C=T$, equation (2) degenerates to (1).

Although not the only yield criterion proposed to account for anisotropy, the one suggested by Hill [24] is certainly the most widely used. It is a modification of equation (1) and may be expressed as,

$$
H\left(\sigma_{1}-\sigma_{2}\right)^{2}+F\left(\sigma_{2}-\sigma_{3}\right)^{2}+G\left(\sigma_{3}-\sigma_{1}\right)^{2}=1
$$

where $H, F$ and $G$ are parameters that characterize a particular state of anisotropy. Because it is again assumed that pressure effects upon yielding are negligible and the tensile and compressive yield strengths in any direction are equivalent, this criterion is unsuitable for general use with anisotropic polymers for reasons discussed earlier.

As equation (1) was modified to (2) for use with isotropic polymers, equation (3) was modified for use with anisitropic polymers [15]. The result is,

$$
H\left(\sigma_{1}-\sigma_{2}\right)^{2}+F\left(\sigma_{2}-\sigma_{3}\right)^{2}+G\left(\sigma_{3}-\sigma_{1}\right)^{2}+K_{1} \sigma_{1}+K_{2} \sigma_{2}+K_{3} \sigma_{3}=1 .
$$

All six coefficients of the stress terms are defined in terms of $C$ and $T$ under the conditions discussed after equation (2) above. Just as it is necessary, for example, to define the constant in equation (1) in terms of measured properties ( $C$ or $T$ for the isotropic case), certain property values must be obtained to define the various parameters in equation (4). These are the tensile and compressive yield strengths in the reference directions which are assumed to coincide with the principal axes of anisotropy (i.e. $T_{1}, T_{2}, T_{3}, C_{1}, C_{2}$ and $C_{3}$ respectively). If the condition of anisotropy is symmetric about the major axis of orientation, which is shown as a reasonable assumption [16], the parameters in equation (4) become,

$$
\begin{gathered}
H+G=\frac{1}{C_{1} T_{1}}, F+H=G+F=\frac{1}{C_{2} T_{2}}=\frac{1}{C_{3} T_{3}} \\
K_{1}=\frac{C_{1}-T_{1}}{C_{1} T_{1}}, K_{2}=K_{3}=\frac{C_{2}-T_{2}}{C_{2} T_{2}}=\frac{C_{3}-T_{3}}{C_{3} T_{3}}
\end{gathered}
$$

+Besides obtaining two such lines, Pae [17] also determined the effect of pressure upon the shear yield strength; this requires specialized equipment. It was from these three discrete lines that he developed yield surfaces. 
again noting that all $C$ terms are absolute values (i.e. $C_{1}=\left|C_{1}\right|$ ). With rotational symmetry, as assumed, $T_{2}=T_{3}$ and $C_{2}=C_{3}$ but $C_{2} \neq T_{2}$.

A more useful form of equation (4) can be developed for the type of experiments involved in this paper. When an all around pressure $\boldsymbol{P}$ is initially applied to a test specimen, the three principal stresses equal $-\boldsymbol{P}$. As a load, either tensile or compressive is then applied in one reference direction, the stress state at any instant may be described by,

$$
\sigma_{1}=S-P, \sigma_{2}=\sigma_{3}=-P
$$

where $S$ is the stress due to the applied load. Tensile loading will cause eventual yielding when a stress, $S_{t}$, reaches a critical value; $S_{\mathrm{c}}$ is the analogous stress to induce compressive yielding.

Now using equations (5) and (6), (4) reduces to,

$$
H\left(\sigma_{1}-\sigma_{2}\right)^{2}+G\left(\sigma_{3}-\sigma_{1}\right)^{2}+K_{1} \sigma_{1}+2 K_{2} \sigma_{2}=1 .
$$

Further simplification results by defining parameters,

$$
A=C_{1} / T_{1} \text { and } R=S / T_{1} .
$$

Introducing equations (5), (6) and (8) into (7) then gives,

$$
R=-\frac{1}{2}(A-1) \pm \frac{1}{2}\left[(A+1)^{2}+4 A P K_{1}+8 A P K_{2}\right]^{1 / 2}
$$

If isotropy prevails, $K_{1}=K_{2}$ and equation (9) reduces to the form used in an earlier study [13] that considered pressure effects on the yield behavior of isotropic polymers.

After $C_{1}, C_{2}, T_{1}$ and $T_{2}$ are obtained at atmospheric pressure, the parameters $A, K_{1}$ and $K_{2}$ can be calculated via equations (5) and (8). Then the two roots of equation (9) may be calculated for specific values of $P$; the positive root, $R_{t}$ predicts the tensile yield strength, $S_{t}$, at that pressure from $S_{t}=R_{t} T_{1}$. Similarly, the compressive yield strength comes from $S_{c}=R_{c} T_{1}$ where $R_{c}$ is the negative root. Thus the effect of pressure upon the yield strengths is predicted as a function of specific property values determined at atmospheric pressure.

\section{PREPARATION OF HIGHLY ORIENTED PC}

To insure a large and consistent degree of anisotropy, the method described in an earlier issue of this journal[15] was used; only the major points are repeated here. Solid bars of PC, having a starting diameter of $54 \mathrm{~mm}$, were used to machine large tensile type specimens having an initial gage dia. of $34 \mathrm{~mm}$ and length of $80 \mathrm{~mm}$. These were then pulled until a stable neck formed and propagated for a length of $100 \mathrm{~mm}$. This highly oriented neck displayed a consistent diameter of $26 \mathrm{~mm}$ (i.e. a true strain of 0.53 ) for all specimens and it is noted that two different original bars were used. From these results it was concluded that the "as received" bars were isotropic in a macroscopic sense and any structural variations across the necked or oriented section were minimal. Both of these assumptions have been fully supported by the findings in an earlier and more thorough investigation [16].

From the oriented material both tension and compression specimens were machined from locations illustrated in Fig. 1. The tensile specimens had a gage length of $25 \mathrm{~mm}$ and dia. of $4.75 \mathrm{~mm}$; all compression specimens were solid, right, circular cylinders of $9 \mathrm{~mm}$ dia. and $18 \mathrm{~mm}$ height. These various specimens were used to determine the basic values of $T_{1}, C_{1}$ and $C_{2}$. Since the diameter of the neck was too small to provide tensile specimens from which $T_{2}$ could be found, two thin-walled tubes were machined from this section. As discussed in detail elsewhere $[15,16,20]$, the hoop yield strength was obtained by conducting an "open-ended" tube test which produced the equivalence of $T_{2}$. A number of tension and compression specimens were machined from locations shown in Fig. 1 to the dimensions mentioned above. These were used to determine the values of yield strength at various hydrostatic pressures; only tests in the 1 direction were so conducted.

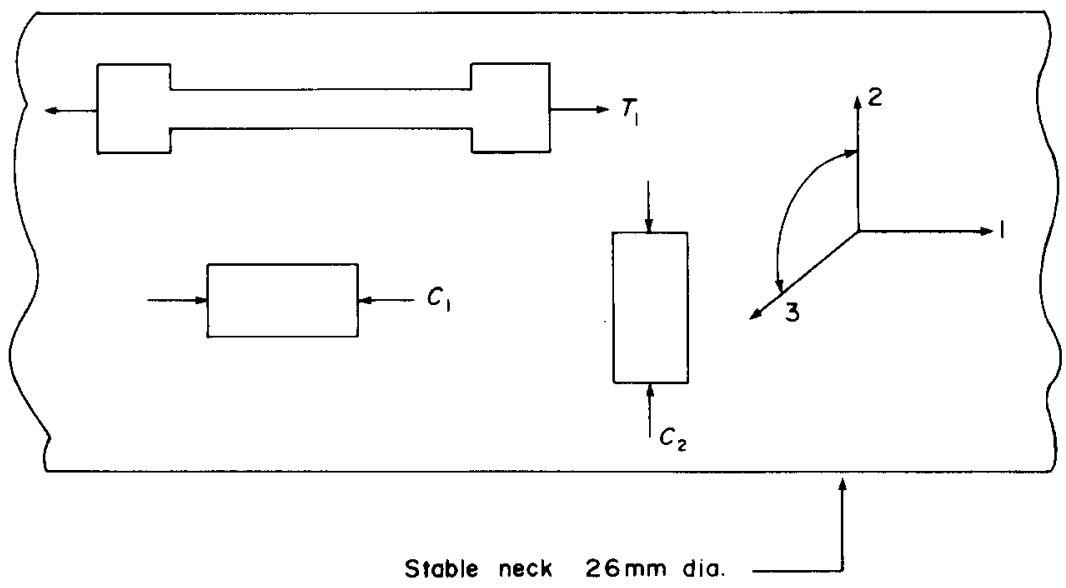

FIG. 1. Orientation of test specimens made from the anisotropic stable neck. 


\section{EXPERIMENTAL RESULTS}

Both tensile and compressive tests were conducted at atmospheric pressure using an Instron machine at the University of Michigan and the pressure machine at Rutgers University. All load-extension or load-compression data were reduced to pertinent values of true stress and true strain; the loading rate was $8.33 \mu \mathrm{m} \mathrm{sec}^{-1}$ throughout. The value for $C_{1}$ ranged from $7700 \mathrm{psi}(53.1 \mathrm{MPa})$ to $7760 \mathrm{psi}(53.5 \mathrm{MPa})$ and the average of $7730 \mathrm{psi}(53.3 \mathrm{MPa})$ was used in later calculations. An average of $8735 \mathrm{psi}(60.2 \mathrm{MPa})$ was found for $C_{2}$, the extreme range being from 8650 to $8820 \mathrm{psi}$.

The values for $T_{1}$ were found to be $1215 \mathrm{psi}(8 \cdot 38 \mathrm{MPa})$ higher when measured with the pressure machine than those obtained from the Instron. This is pursued further at a later place in this paper; for now it will suffice to state that the average value for $T_{1}$ was $10,715 \mathrm{psi}(73.9 \mathrm{MPa})$. From the open ended tube tests a corrected average value of $T_{2}$ was taken as $6715 \mathrm{psi}(46.3 \mathrm{MPa})$; this is also discussed later.

Using the values indicated above, the variations in $S_{t}$ and $S_{c}$, related to equation (9), were predicted as a function of hydrostatic pressure up to a level of $80 \mathrm{ksi}(0.55 \mathrm{GPa})$. The results are shown by the two solid lines on Fig. 2. Subsequent experiments were performed on the pressure machine using pressures of 20, 40, 60 and $80 \mathrm{ksi}$ (see SI units on Fig. 2), both the tensile and compressive yield strengths being determined at each discrete pressure. Individual results are plotted on Fig. 2 and a promising correlation is shown.

\section{(1) Definition of yield strength}

\section{DISCUSSION}

In this study every "yield strength" was determined using a $1 \%$ off set; this is often termed a proof stress. Although the maximum load, prior to the formation of a stable neck, is often taken as the yield load, this approach could not be followed consistently in this study. For example, tests run on tensile specimens made for oriented material in the " 1 " direction displayed no such maximum load. For consistency, the off set method was used and although a $1 \%$ value is certainly arbitrary, in those tests where a maximum load was observed it was very close to the value coinciding with this offset; for that reason the $1 \%$ value was chosen.

\section{(2) Determining compressive yield strength}

When a direct compression test is run on a typical testing machine (e.g. an Instron machine) it is essential to account for the elastic effects of the machine and, especially, the load cell if cross-head displacement is to be used to determine the actual strain experienced by the specimen. It might be assumed that because polymers possess a much lower elastic modulus than the typical machine components that any such effects are negligible; this is an erroneous assumption. One method used to overcome any error is to apply a compressive load to the system without using a test specimen; this provides a load-displacement plot as shown by $\boldsymbol{A}$ in Fig. 3. Now when a test specimen is subjected to compressive loading, the resulting plot includes not only the displacement of the specimen but also the contribution of the machine and load cell deflections; a curve such as $B$ results in Fig. 3. The correct load-displacement behavior of the test specimen results by subtracting the effects of $A$ from $B$ and the proper curve is shown as $C$. If such a correction is ignored, large errors occur in the early stages of deformation and the stress-strain plot that results will lead to substantial errors in values of the elastic modulus determined from such a plot. It is our opinion that many of the quoted numerical values of elastic moduli of polymers, as influenced by pressure, are open to question since this type of correction is seldom, if ever, mentioned. In situations where the stiffness correction factor, i.e. curve $A$ on Fig. 3, is not linear, values of yield strength based upon an off set method are also open to question. For all values of compressive yield strength quoted in this paper, a pertinent stiffness correction factor, related to both the Instron and pressure machines, was used.

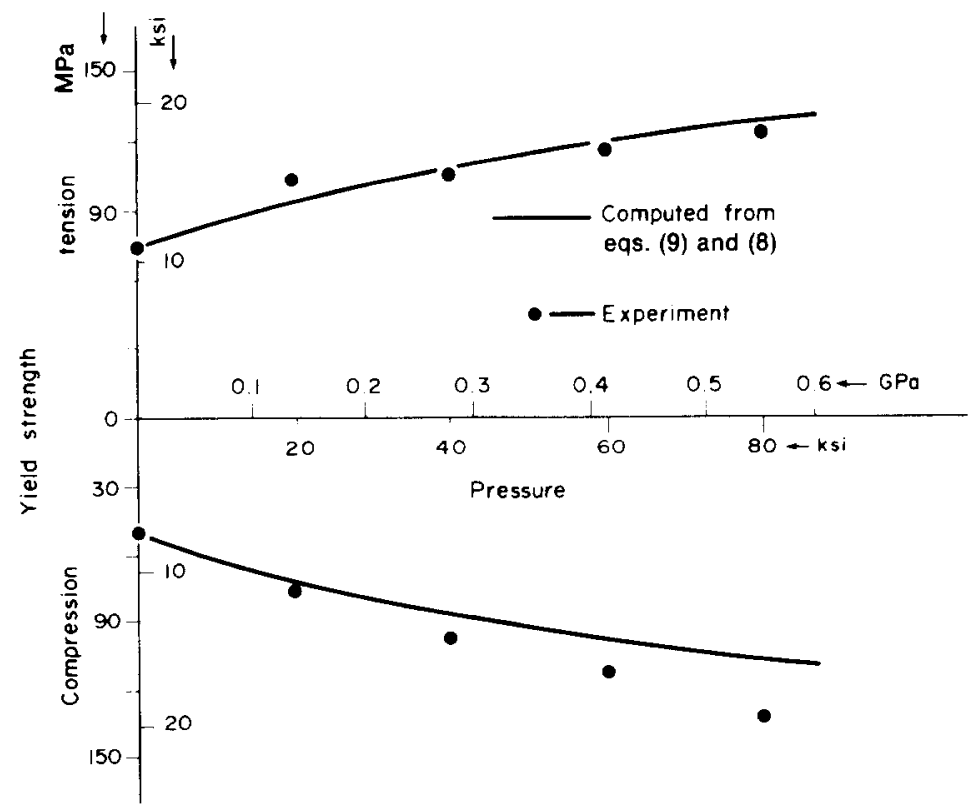

Fig. 2. Comparison of measured values of tensile and compressive yield strength with predictions based upon a yield criterion for anisotropic and pressure dependent solids. 


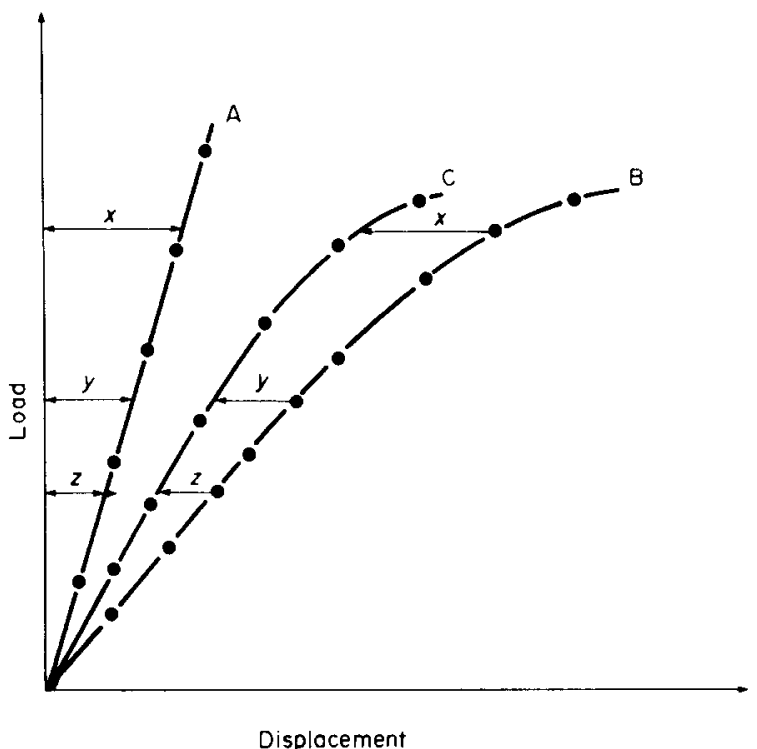

FiG. 3. Method for correcting compressive load-displacement data to account for all elastic deflections other than the test specimen.

(3) Determining tensile yield strength

By using stiffness corrections for both machines, the variation in compressive yield strengths was found to be minor as indicated by the $C_{1}$ and $C_{2}$ values quoted earlier. Tensile testing presents a more involved experimental problem. Using the Instron and an extensometer adapted to the gage length of a tensile specimen, the stress-strain behavior shown as curve $A$ on Fig. 4 resulted. This must be accepted as correct since any displacements outside of the gage length do not influence such results. It is when the pressure machine is used for tensile testing that problems arise. Because of its design, extensions of the test portion of the gage section cannot be easily measured with this machine. Common practice is to measure the external displacement in line with the direction of load application and assume that this entire displacement occurred within the gage length only. This introduces a potentially serious error if such data are converted to provide a stress-strain plot! Curves $B$ and $C$ on Fig. 4 illustrate this point, their difference arising from the use of two specimens identical in all respects except for the starting gage length. Curve $B$ resulted when a specimen having a $25 \mathrm{~mm}$ gage length was used; with $C$, this length was $12.5 \mathrm{~mm}$. Neither curve duplicates the correct one, i.e. $A$, the basic error arising primarily from incorrect strain calculations. By assuming the total external displacement results from displacements of the gage length alone, one ignores the elastic displacement of the threaded ends

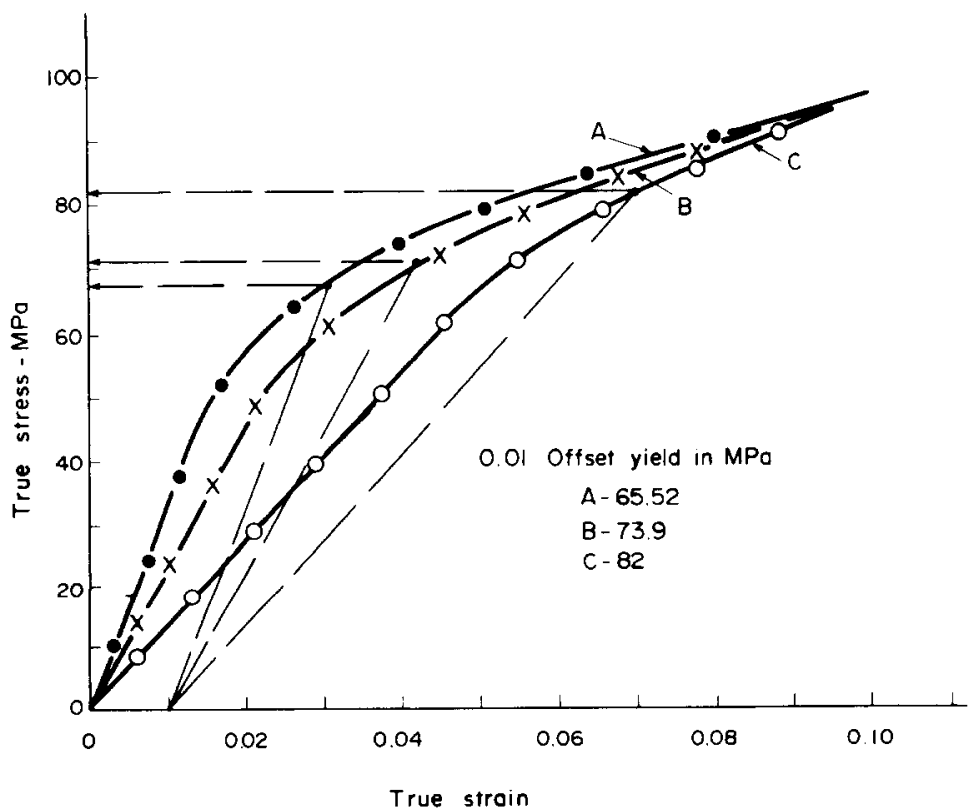

FIG. 4. True stress-true strain curve from Instron test $(A)$ and two tests using pressure unit $(B$ and $C$ ) where two different starting gage lengths were used. 
and other such factors. Thus, the computed strains are always too large. Such errors may be reduced, but not eliminated, by using specimens of longer starting gage lengths. For the same external displacement, i.e. $\Delta l$, the computed strain, $\Delta l / l_{0}$ or $\ln \left(l / l_{0}\right)$, will be smaller as $l_{0}$ increases. This is shown by curves $B$ and $C$ on Fig. 4 and is analogous to the concept of Cook and Larke [25] to eliminate end effects during compression testing. If, in effect, tensile specimens of infinite gage length were used, this problem would be resolved; of course, this is a practical impossibility.

This presented a still unresolved quandry when tensile information was to be obtained with the pressure machine, since all such tests under pressure had to be conducted on that device. The value of $T_{1}$ quoted earlier (i.e. $10,715 \mathrm{psi}$ or $73.9 \mathrm{MPa}$ ), and used in connection with the plot on Fig. 2 , was the average value found with the pressure machine using specimens of $25 \mathrm{~mm}$ gage length. This was $1215 \mathrm{psi}(8.38 \mathrm{MPa})$ higher than the average and correct value found with the Instron. Values for $T_{2}$ could not be obtained with the pressure device as explained earlier, the only recourse being the open-ended tube test; this was measured as $5500 \mathrm{psi}(37.9 \mathrm{MPa})$. It seems reasonable to conclude that if $T_{2}$ could be obtained from the pressure machine, it would also be higher than that using tube tests so a modified value for $T_{2}$ seemed necessary. Although certainly open to question, the most direct approach was to increase the tube value by the same differential displayed between the Instron and pressure machines for $T_{1}$. This led to a modified value for $T_{2}$ of $6715 \mathrm{psi}(46.3 \mathrm{MPa})$; it is this value that was then used in connection with the plotted lines on Fig. 2.

As a final point, the stiffness correction factor for compression tests was found to be independent of pressures up to $100 \mathrm{ksi}(689 \mathrm{MPa})$ and the application of this factor led to stress-strain behavior, from which a correct elastic modulus was determined for such loading. Since equivalent corrections were not possible with the tensile data obtained under pressure, it was assumed that the tensile and compressive moduli were equivalent; Woodliff [26] has shown this is quite reasonable. By adjusting all tensile data to produce the same modulus displayed in compression, a corrected tensile stress-strain curve was determined. From such curves, the $1 \%$ off set yield strengths were then found at each test pressure.

\section{CONCLUSIONS}

A proposed yield criterion for use with anisotropic and pressure dependent polymers has been compared with experimental findings and the results are in good agreement. Since only one test material was used, further studies involving other anisotropic polymers should be pursued to determine if this criterion provides general application. Certain comments have been made regarding the problems involved in determining the correct stress-strain behavior when pressure machines are used. Tensile testing is especially difficult in this regard and definitely warrants further study.

Acknowledgements - This material is based upon work supported by the National Science Foundation under Grant DMR-74-10355. That assistance is gratefully acknowledged here. Professor K. D. Pae provided useful comments and permitted us to conduct experiments on his high pressure machine; we thank him for his interest in this study. Finally, Prof. W. F. Hosford and S. B. Mathur made a number of helpful comments and suggestions; their interest is also deeply appreciated.

\section{REFERENCES}

1. J. A. Sauer, K. D. Pae and S. K. Bhateja, J. Macromol. Sci.-Phys. B8(3, 4), 631 (1973).

2. K. D. PAE and S. K. Bhateja, J. Macromol. Sci.-Rev. Macromol. Chem. C13(1), 1 (1975).

3. S. K. Bhateja and K. D. PAE, J. Macromol. Sci.-Rev. Macromol. Chem. C13(1), 77 (1975).

4. A. W. Christiansen, E. Baer and S. V. RadCliffe, Phil. Mag. 24, 451 (1971).

5. D. Sardar, S. V. RadCliffe and E. Baer, Polym. Engng Sci. 8, 290 (1968).

6. K. D. PAe and D. R. Mears, Polym. Lett. 6, 269 (1968).

7. G. Biglione, E. Baer and S. V. RadCliffe, Proc. 2nd Int. Conf. on Fract. Bristol, England (1969).

8. D. R. Mears, K. D. Pae and J. A. Sauer, J. Appl. Phys. 40, 4229 (1969).

9. S. B. Ainbinder, M. G. LaKa and I. Yu Maiors, Mekh. Polim. 1, 65 (1965).

10. W. A. SPITZIG, R. J. Sober and O. Richmond, Acta Met. 23, 885 (1975).

11. W. A. SpITZig, R. J. SOBER and O. Richmond, Met. Trans. A. 7A, 1703 (1976).

12. D. C. DRUCKER, Met. Trans. 4, 667 (1973).

13. R. M. Caddell, R. S. Raghava and A. G. Atkins, Mat. Sci. and Engng 13, 113 (1974).

14. R. M. Caddell, R. S. Raghava and A. G. Atkins, J. Mat. Sci. 8, 1641 (1973).

15. R. S. RaGHAVA and R. M. CaḊDELL, Int. J. Mech. Sci. 16, 789 (1974).

16. R. M. CADDELL and A. R. WoOdLIFF, J. Mat. Sci. 12, 2028 (1977).

17. K. D. PAE, J. Mat. Sci. 12, 1209 (1977).

18. J. Sauer, D. R. Mears and K. D. PaE, Europ. Poly. J. 6, 1015 (1970).

19. R. von MISES, Gott. Nach., Math. Phys. Klasse, 583 (1913).

20. R. S. Raghava, Ph.D. Diss., University of Michigan, Ann Arbor, Michigan (1972).

21. F. Stassi D'Alia, Meccanica, 3, 178 (1967).

22. R. E. ELY, Polym. Engng Sci. 7, 40 (1967).

23. W. F. HosforD, Met. Engng Quart. 6, 13 (1966).

24. R. Hill, Plasticity, p. 315. Clarendon Press, Oxford (1950).

25. M. COOK and E. C. LARKE, J. Inst. Metals, 71, 371 (1945).

26. A. R. Woodliff, Ph.D. Diss., University of Michigan, Ann Arbor, Michigan (1978). 